\title{
Medication overuse headache: a critical review of end points in recent follow-up studies
}

\author{
Knut Hagen · Rigmor Jensen · Magne Geir Bøe • \\ Lars Jacob Stovner
}

Received: 22 February 2010/ Accepted: 25 April 2010/Published online: 16 May 2010

(C) Springer-Verlag 2010

\begin{abstract}
No guidelines for performing and presenting the results of studies on patients with medication overuse headache $(\mathrm{MOH})$ exist. The aim of this study was to review long-term outcome measures in follow-up studies published in 2006 or later. We included MOH studies with $>6$ months duration presenting a minimum of one predefined end point. In total, nine studies were identified. The 1,589 MOH patients (22\% men) had an overall mean frequency of 25.3 headache days/month at baseline. Headache days/month at the end of follow-up was reported in six studies (mean 13.8 days/month). The decrease was more pronounced for studies including patients with migraine only ( -14.6 days/month) compared to studies with the original diagnoses of migraine and tension-type headache ( -9.2 days/month). Six studies reported relapse rate (mean of $26 \%$ ) and/or responder rate (mean of $28 \%$ ). Medication days/month and change in headache index at the end of follow-up were reported in only one and two of nine
\end{abstract}

\author{
K. Hagen · L. J. Stovner \\ Department of Neuroscience, Faculty of Medicine, \\ Norwegian University of Science and \\ Technology, Trondheim, Norway \\ K. Hagen $(\varangle) \cdot$ L. J. Stovner \\ Section of Neurology, Department of Neurology, \\ Norwegian National Headache Centre, St. Olavs Hospital, \\ 7006 Trondheim, Norway \\ e-mail: knut.hagen@ntnu.no \\ R. Jensen \\ Department of Neurology, Danish Headache Centre, \\ University of Copenhagen, Glostrup Hospital, \\ Glostrup, Denmark \\ M. G. Bøe \\ Department of Neurology, Sørlandet Hospital, \\ Kristiansand, Norway
}

studies, respectively. The present review demonstrated a lack of uniform end points used in recently published follow-up studies. Guidelines for presenting follow-up data on $\mathrm{MOH}$ are needed and we propose end points such as headache days/month, medication days/month, relapse rate and responder rate defined as $\geq 50 \%$ reduction of headache frequency and/or headache index from baseline.

Keywords Medication overuse headache - Follow-up · Outcome parameters $\cdot$ Relapse rate $\cdot$ Responders

\section{Introduction}

Worldwide, approximately $1-2 \%$ of the adult general population suffers from chronic headache ( $\geq 15$ days/ month) combined with medication overuse [1-6]. The optimal method of treating the many patients with medication overuse headache $(\mathrm{MOH})$ is still controversial, mainly due to lack of placebo-controlled, double-blind, randomized clinical trials [7]. No established consensus for treatment strategies exists for patient with $\mathrm{MOH}$. As a consequence, the therapeutic strategies for the acute phase of detoxification and the use of preventive treatment differ widely between studies [8].

Most previous follow-up studies of patients with $\mathrm{MOH}$ are case series and non-randomized studies, which are difficult to interpret. The lack of high-quality studies makes it difficult to draw firm conclusions regarding the management of $\mathrm{MOH}$ [8]. During the last two decades, several guidelines for controlled treatment trials for patients with migraine and tension-type headache have been published [9-11], also emphasizing the need of uniform end points. However, no guidelines on performing and presenting the result of studies on $\mathrm{MOH}$ patients exist. Due to the lack of 
guidelines, it may be of relevance to review the type of end points published in recent follow-up studies of $\mathrm{MOH}$ patients.

The aim of this study was to analyze and summarize the long-term outcome measures of $\mathrm{MOH}$ patients included in follow-up studies published in 2006 or later.

\section{Methods}

Follow-up studies of patients with $\mathrm{MOH}$ published in English were identified through PubMed by searching for relevant publications between January 2006 and December 2009. We used the search terms "medication overuse headache" combined with "follow-up." References listed in relevant publications were also examined. All studies were first screened for various aspects of methodology and design, and type of content, to select studies of interest for our purpose. Studies on $\mathrm{MOH}$ patients who underwent one type of intervention were labeled case series, whereas studies including at least two types of interventions were labeled controlled studies. We included studies fulfilling the following four minimum criteria:

(1) published in 2006 or later. Recently published studies were preferred because the majority of these studies included patients fulfilling the Headache Classification Committee of the International Headache Society from 2004 (ICDH-2) or later modified versions [1214];

(2) >6 months' duration of follow-up;

(3) at least two of the following characteristics mentioned explicitly at baseline: primary headache type, headache days/month and/or medication days/month;

(4) at least one of the following end points mentioned explicitly at follow-up: headache days/month, reduction of headache frequency from baseline, medication days/month, reduction of medication days from baseline, responder rate defined as the proportion of individuals with $\geq 50 \%$ reduction of headache frequency from baseline [10], or relapse rate defined as the proportion of individuals with the diagnosis of $\mathrm{MOH}$ at the end of follow-up among subjects with "successful withdrawal." In this context patients with successful withdrawal were defined as those not overusing medication after the withdrawal period, regardless of whether they experienced reduction of headache frequency or not.

\section{Statistics}

Mean values of the total group of participants were presented if available. If mean values of, for e.g., three subgroups were presented, mean values for the total group were calculated by the following formula: (days/month $\times$ number of participants in group $\mathrm{A}+$ days/month $\times$ number of participants in group $\mathrm{B}+$ days/month $\times$ number of participants in group C) divided by total number of participants in group A, B and C. Mean reduction of headache days/month (as percentage) from baseline was also calculated.

\section{Results}

Initially, 33 studies were identified by the PubMed search. This was reduced to 28 unique studies after removing multiple publications based on the same patients [15-19]. Overall, nine follow-up studies, all clinic-based, fulfilled the four criteria of the present review. To optimize comparisons between studies, 12 months follow-up data were preferred in one study, although 3- and 5-year follow-up data were also available [19]. Five studies included only $\mathrm{MOH}$ patients who had had migraine before developing $\mathrm{MOH}$, whereas the remaining four studies also included patient who had had TTH (Table 1) [15, 20-27]. Four follow-up studies were case series without controls [20-22, 26]: one retrospective [20] and three prospective studies $[21,22,26]$. The remaining five studies were categorized as controlled studies with $(n=3)$ [23-25] or without confirmed randomization $(n=2)[15,27]$ (Table 1$)$.

Headache diary was used in five studies during the follow-up [20, 22-25] and most likely used, although not explicitly stated, in two additional studies $[15,27]$. In eight studies (Table 1) [15, 21-27], 12-month follow-up data were available. Two Italian studies used the criteria for chronic migraine proposed by Silberstein and Lipton in 2000 [15, 22], whereas the remaining patients fulfilled the Headache Classification Committee of the International Headache Society from 2004 (ICDH-2) [20, 23, 25, 27] or later modified versions [21, 24, 26]. Although the specific treatment protocol differed widely between the nine studies, all studies included abrupt withdrawal as a part of the protocol (Table 2). Prophylactic treatment was initiated during the first week in the majority of studies (range 1-90 days) (Table 2).

The 1,589 $\mathrm{MOH}$ patients (22\% men) included in the nine different studies had an overall mean of 25.3 headache days per month (range 22.5-27.0) at baseline. Headache days at the end of follow-up were reported in six studies ( $n=582$, mean follow-up duration 11.3 months, mean -13.8 days $/$ month $=45 \%$ decrease from baseline) $[15$, 20, 22, 24, 25, 27]. The mean decrease was more pronounced for the studies including patients with migraine only ( $n=290$, mean follow-up duration 12 months, -14.6 days $/$ month $=56 \%$ decrease from baseline) $[15,22$, 27] than among those with migraine and TTH $(n=29$, 
Table 1 Study characteristics at baseline

\begin{tabular}{|c|c|c|c|c|c|c|}
\hline $\begin{array}{l}\text { Study, } \\
\text { reference }\end{array}$ & Country & $\begin{array}{l}\text { Number included } \\
(\% \text { men })\end{array}$ & $\begin{array}{l}\text { Age group } \\
\text { (range) }\end{array}$ & $\begin{array}{l}\text { TTH } \\
\text { included }\end{array}$ & $\begin{array}{l}\text { Duration } \\
\text { (months) }\end{array}$ & Design \\
\hline $2006[20]$ & Denmark & $337(27)$ & $17-86$ & Yes & 8 & Retrospective case series \\
\hline 2007 [21] & Serbia & 240 (24) & $17-76$ & Yes & 12 & Case series \\
\hline $2007[22]$ & Italy & $106(18)$ & $19-71$ & No & $12^{\mathrm{f}}$ & Case series \\
\hline 2008 [23] & Italy & $118(16)$ & - & No & 12 & Randomized controlled $^{\mathrm{a}}$ \\
\hline $2009[24]$ & Norway & $61(39)$ & $18-70$ & Yes & 12 & Randomized controlled ${ }^{\mathrm{b}}$ \\
\hline 2009 [25] & Norway & $100(26)$ & $18-70$ & Yes & 12 & Double-blind $\mathrm{RCT}^{\mathrm{c}}+$ randomized controlled ${ }^{\mathrm{c}}$ \\
\hline 2009 [26] & Italy & $215(19)$ & - & No & 12 & Case series \\
\hline 2009 [27] & Italy & $93(-)$ & - & No & 12 & Controlled $^{\mathrm{d}}$ \\
\hline 2009 [15] & Italy & $260(-)$ & - & No & 12 & Controlled $^{\mathrm{e}}$ \\
\hline
\end{tabular}

a Three groups: advice alone versus structured detoxification as in- or outpatient

b Three groups: prophylactic treatment from the start without detoxification versus standard outpatient detoxification program without prophylactic treatment from the start versus no specific treatment (controls)

c Two groups: placebo-controlled, double-blind randomized controlled trial (RCT) evaluating efficacy of prednisolone on withdrawal headache followed by an controlled design evaluating long-term outcome of patients treated by neurologist versus GP

d Two groups; randomization not stated: behavioral plus pharmacological treatment versus pharmacological treatment only

e Two groups, randomization not stated: inpatient versus day-hospital withdrawal treatment

${ }^{\mathrm{f}}$ Data on 3 and 5 years follow-up also available

Table 2 Initial treatment strategies

\begin{tabular}{llllll}
\hline Study, reference & Abrupt withdrawal? & In- or outpatient & Days at hospital & Infusion therapy? & Onset of preventive drugs \\
\hline $2006[20]$ & Yes & Outpatient & 0 & No & After 2 months \\
$2007[21]$ & Yes & In- or outpatient & 8 & Yes & During the first weeks? \\
$2007[22]$ & Yes & Inpatient & 10 & Yes & Day 6 \\
$2008[23]$ & Yes & Inpatient & 8 & Yes & Day 1 \\
$2009[24]$ & Yes & Outpatient & 0 & No & Day 1 or after 3 months \\
$2009[25]$ & Yes & Inpatient & 3 & No & After 1 months \\
$2009[26]$ & Yes & Inpatient & 10 & Yes & Day 4 or 5 \\
$2009[27]$ & Yes & Day patient & 5 & Yes & Day 5? \\
$2009[15]$ & Yes & In- or day patient & 7 & Yes & Day 6
\end{tabular}

${ }^{a}$ Abrupt withdrawal in one out of three groups

b Incomplete information

mean follow-up duration 10.7 months, -9.2 days/month $=$ $36 \%$ decrease from baseline) $[20,24,25]$. The response rate and relapse rate were reported in only three [20, 24, $25]$ and four studies [21, 23, 25, 26], respectively, with a mean of 28 and $26 \%$ (Table 3). Medication days/month at the end of follow-up was reported in one study only [24], whereas two studies revealed information about change in the headache index at follow-up compared to baseline (data not given) $[24,25]$.

\section{Discussion}

Study design and treatment strategy differ widely between the studies, and direct comparisons should be done with caution. However, in a recent review it was suggested that differences in withdrawal therapy strategy seem to have no major impact on long-term outcome [8]. If true, an overall $45 \%$ reduction in headache days and a $28 \%$ response rate during 1 year should be expected for the group of $\mathrm{MOH}$ patients in an open-labeled setting. However, the tendency of remission or worsening over time must be taken into account since these factors may overestimate the effect of treatment [28]. It should be emphasized that the lack of control group in all studies (except one) increases the risk that these results may be explained, at least in part, by the natural history or regression from the mean. These flaws may be avoided in randomized, double-blind, placebocontrolled studies. In one placebo-controlled study evaluating the efficacy of topiramate without withdrawal, a more 
Table 3 End points used in nine follow-up studies

\begin{tabular}{|c|c|c|c|c|c|c|c|c|}
\hline $\begin{array}{l}\text { Study, } \\
\text { reference }\end{array}$ & $\begin{array}{l}\text { Number of } \\
\text { dropouts }(\%)\end{array}$ & $\begin{array}{l}\text { Headache days/ } \\
\text { month baseline }\end{array}$ & $\begin{array}{l}\text { Duration of } \\
\text { follow-up } \\
\text { (months) }\end{array}$ & $\begin{array}{l}\text { Headache } \\
\text { days/month } \\
\text { Follow-up }\end{array}$ & $\begin{array}{l}\text { Medication days/ } \\
\text { month baseline }\end{array}$ & $\begin{array}{l}\text { Medication days/ } \\
\text { month follow-up }\end{array}$ & $\begin{array}{l}\text { Response rate } \\
\text { Number }(\%)\end{array}$ & $\begin{array}{l}\text { Relapse rate } \\
\text { Number }(\%)\end{array}$ \\
\hline 2006 [20] & $162 / 337(48)$ & 27 & 8 & $15^{\mathrm{e}}$ & - & - & $67 / 337^{\mathrm{b}}(20)$ & - \\
\hline 2007 [21] & - & 24.6 & 12 & - & - & - & - & $95 / 240(39.6)$ \\
\hline 2007 [22] & $22 / 106(21)$ & 26.1 & 12 & 11.5 & - & - & - & - \\
\hline 2008 [23] & $37 / 118(31)$ & 26.8 & 12 & - & - & - & - & $17 / 83(20.5)$ \\
\hline 2009 [24] & $5 / 61(8)$ & 24.1 & $12^{\mathrm{b}}$ & $17.1^{\mathrm{e}}$ & 23.2 & 8.0 & $14 / 41^{\mathrm{c}}(34)$ & - \\
\hline 2009 [25] & $20 / 100(20)$ & 25.4 & 12 & $16.7^{\mathrm{e}}$ & - & - & $27 / 93^{\mathrm{d}}(29)$ & $16 / 80(20)$ \\
\hline 2009 [26] & $43 / 215(20)$ & 22.5 & 12 & - & 21.6 & - & - & $38 / 172(22)$ \\
\hline 2009 [27] & $36 / 93(39)$ & 26.2 & 12 & 12.0 & - & - & - & - \\
\hline 2009 [15] & $111 / 260(43)$ & 25.3 & 12 & 10.4 & - & - & - & - \\
\hline Number & 8 & 9 & 9 & 6 & 2 & 1 & 3 & 4 \\
\hline Mean & 29 & 25.3 & 11.6 & 13.8 & - & - & 28 & 25.5 \\
\hline
\end{tabular}

${ }^{a}$ Calculation of response rate (proportion of individuals with $\geq 50 \%$ reduction in headache frequency from baseline) differ between the three studies with respect to (a) number of participants included in the analysis and (b) time interval before evaluation

b Analysis of 337 participants (all included) evaluated after a mean of 8 months

c Analysis of 41 individuals evaluated after 12 months (20 controls excluded because of 5 months follow-up duration)

d Analysis of 93 individuals evaluated 3 months after withdrawal

e TTH patients included

moderate decrease in headache days/month (approximately $25 \%$ ) was found among $\mathrm{MOH}$ patients with chronic migraine [29].

The most important finding in the present review was a lack of uniform end points used in the nine follow-up studies. Headache days/month was the most commonly presented, followed by relapse rate and/or responder rate. Surprisingly, only two studies explicitly reported medication days/month at baseline $[24,26]$ and only one study did so at the end of follow-up [24], although this was crucial when considering the diagnosis of $\mathrm{MOH}$ and relapse rate. Although not clearly stated, one may assume that most studies had collected information about medication days/month at baseline according to $\mathrm{MOH}$ diagnosis based on ICDH-2 or later modified versions. Less informative than medication days/ month is the total number of medication doses consumed per month that was reported in four studies [15, 22, 23, 27]. The definition of responders varied between studies. In the study by Rossi et al. [23], responders were defined as those who had headache $<15$ days per month and intake of symptomatic medication $<10$ days/month 2 month after withdrawal treatment. However, using this definition (although it fulfills the ICDH-2 criteria), patients may be defined as responders even with a minor reduction in headache and intake of medication. In our opinion defining a responder as a patient with $\geq 50 \%$ reduction of headache frequency from baseline is more informative, although other definitions, e.g., $\geq 25 \%$ reduction of headache frequency from baseline, can be clinically meaningful for $\mathrm{MOH}$ patients [10]. Furthermore, for MOH patients headache index rather than headache days may more correctly reflect the total suffering [11]. Two studies had included such data based on frequency, duration and intensity [24] or frequency and intensity (named mean headache in the publication) [25] recorded in headache diaries. One may still discuss what the optimal definition of a responder is, but we would favor studies on $\mathrm{MOH}$ treatment that aim at $\geq 50 \%$ reduction in headache index from baseline. This was used as a secondary end point in one study [25]. In accordance, headache index as a primary efficacy measure has been proposed in the revised version of the guidelines for controlled trials of prophylactic drugs in chronic tensiontype headache [11]. However, for headache frequency one should acknowledge that less ambitious definition of end points, e.g., those with $\geq 25 \%$ reduction of headache index from baseline, can be clinically meaningful [10].

In one out of the four studies reporting relapse rates [21, $23,25,26]$, the calculation was restricted to individuals who had an improvement of headache at follow-up after 2 months [23]. However, in the study by Bøe et al. [25], a much lower relapse rate at 12 months follow-up was found among individuals with $\geq 50 \%$ improvement score at 3 months follow-up than among those without such improvement ( 4 vs. 28\%). Thus, a higher relapse rate may be expected if individuals with no or some improvement of headache frequency after successful withdrawal are included in the analysis.

The review demonstrated a lack of uniform end points in recently published follow-up studies of patient with $\mathrm{MOH}$. 
If possible, the evaluation of long-term outcome of patient with $\mathrm{MOH}$ should be based on headache diary information preferentially including data on headache days/month, headache intensity and medication days/month. We recommend that at the end of follow-up, minimum headache days/month and medication days/month are included, since these end points are needed to calculate relapse rate. In addition, we recommend that headache intensity and attack duration are included and that the responder rate is defined as $\geq 50 \%$ reduction of headache frequency and/or headache index from baseline. However, including end points such as $\geq 25 \%$ reduction of headache frequency and/or headache index may add useful information to the final selection of end points that should be preferred in the future. There is a need for increasing awareness of methodological issues in clinical follow-up studies for $\mathrm{MOH}$. Guidelines for $\mathrm{MOH}$ studies including use of end points are needed.

\section{Conflict of interest None.}

\section{References}

1. Zwart JA, Dyb G, Hagen K, Svebak S, Stovner LJ, Holmen J (2004) Analgesic overuse among subjects with headache, neck and low-back pain. Neurology 62:1540-1544

2. Lu SR, Fuh JL, Chen WT, Juang KD, Wang SJ (2001) Chronic daily headache in Taipei, Taiwan: prevalence, follow-up and outcome predictors. Cephalalgia 21:980-986

3. Wang SJ, Fuh JL, Liu CY, Hsu LC, Wang PN, Liu HC (2000) Chronic daily headache in Chinese elderly. Prevalence, risk factors, and biannual follow-up. Neurology 54:314-319

4. Castillo J, Muñoz P, Guitera V, Pascual J (1999) Epidemiology of chronic daily headache in the general population. Headache 39:190-196

5. Colás R, Muñoz P, Temprano R, Gómez C, Pascual J (2004) Chronic daily headache with analgesic overuse: epidemiology and impact on quality of life. Neurology 62:1338-1342

6. Aaseth K, Grande RB, Kværner KJ, Gulbrandsen P, Lundqvist C, Russell MB (2008) Prevalence of secondary chronic headaches in a population-based sample of 30-44-year-old persons. The Akerhus study of chronic headache. Cephalalgia 28:705-713

7. Dodick DW (2006) Clinical practice. Chronic daily headache. N Engl J Med 354:158-165

8. Rossi P, Jensen J, Nappi G, Allena M (2009) The COMOESTAS Consortium. A narrative review on the management of medication overuse headache: the steep road from experience to evidence. J Headache Pain 10:407-417

9. International Headache Society Committee on Clinical Trials in Migraine (1991) Guidelines for controlled trials of drugs in migraine, 1st edn. Cephalalgia 11:1-12

10. Silberstein S, Tfelt-Hansen P, Dodick DW, Limmroth V, Lipton RB, Pascual J, Wang SJ (2008) Task Force of the International Headache Society Clinical Trials Subcommittee. Guidelines for controlled trials of prophylactic treatment of chronic migraine in adults. Cephalalgia 28:484-495

11. Bendtsen L, Bigal ME, Cerbo R, Diener HC, Holroyd K, Lampl C, Mitsikostas DD, Steiner TJ, Tfelt-Hansen P (2009) Guidelines for controlled trials of prophylactic treatment of chronic migraine in adults. Guidelines for controlled trials of drugs in tension-type headache: second edition. Cephalalgia [Epub ahead of print]
12. Headache Classification Committee of International Headache Society (2004) The international classification of headache disorders. 2nd ed. Cephalalgia 24(Suppl 1):1-160

13. Silberstein S, Olesen J, Bousser MG, Diener HC, Dodick D, First M, Goadsby PJ, Göbel H, Lainez M, Lance LW, Lipton RB, Nappi G, Sakai F, Schoenen J, Steiner TS, on behalf of the International Headache Society (2005) The International Classification of Headache Disorders, 2nd Edition (ICHD-II)-revision of criteria for 8.2 Medication-overuse headache. Cephalalgia 25:460-465

14. Olesen J, Bousser MG, Diener HC, Dodck D, First M, Goadsby PJ, Göbel H, Lainez MJA, Lance JW, Lipton RB, Nappi G, Sakai F, Schoenen J, Silbertstein SD, Steiner TJ, Headache Classification Committee (2006) New appendix criteria open for a broader concept of chronic migraine. Cephalalgia 26:742-746

15. Usai S, Grazzi L, D’Amico D, Andrasik F, Bussone G (2009) Psychological variables chronic migraine with medication overuse before and after inpatient withdrawal: results at 1-year follow-up. Neurol Sci 30:S125-S127

16. Usai S, Grazzi L, D’Amico D, Andrasik F, Bussone G (2008) Reduction in the impact of chronic migraine with medication overuse after day-hospital withdrawal therapy. Neurol Sci 29:S176-S178

17. Ghiotto N, Sances G, Galli F, Tassorelli C, Guaschino Sandrini G, Nappi G (2009) Medication overuse headache and applicability of the ICDH-II diagnostic criteria: 1 year follow-up study (care I protocol). Cephalalgia 29:233-243

18. Bøe MG, Salvesen R, Mygland A (2009) Chronic daily headache with medication overuse: predictors of outcome 1 year after withdrawal therapy. Eur J Neurol 16:705-712

19. Andrasik F, Grazzi L, Usai S, Kass S, Bussone G (2009) Disability in chronic migraine with medication overuse: treatment effects through 5 years. Cephalalgia [Epub ahead of print]

20. Zeeberg P, Olesen J, Jensen R (2006) Discontinuation of medication overuse in headache patients: recovery of therapeutic responsiveness. Cephalalgia 26:1192-1198

21. Zidverc-Trajkovic J, Pekmezovic T, Jovanovic Z, Pavlovic A, Mijajlovic M, Radojicic A, Sternic N (2007) Medication overuse headache: clinical features predicting treatment outcome at 1year follow-up. Cephalalgia 27:1219-1225

22. Andrasik F, Grazzi L, Usai S, D’Amico D, Kass S, Bussone G (2007) Disability in chronic migraine with medication overuse: treatment effects at 3 years. Headache 47:1277-1281

23. Rossi R, Faroni JV, Nappi G (2008) Medication overuse headache: predictors and rates of relapse in migraine patients with low medical needs. A 1-year prospective study. Cephalalgia 28:1196-1200

24. Hagen K, Albretsen C, Stovner LJ, Vilming S, Salvesen R, Grønning M, Helde G, Gravdahl G, Zwart JA (2009) Management of probable medication-overuse headache: 1-year randomized prospective multicenter trial. Cephalalgia 29:221-232

25. Bøe MG, Salvesen R, Mygland A (2009) Chronic daily headache with medication overuse: a randomized follow-up by neurologist or PCP. Cephalalgia 9:855-863

26. Sances G, Ghiotto N, Galli F, Guaschino E, Rezzani C, Guidetti V, Nappi G (2009) Risk factors in medication-overuse headache: a 1 year follow-up study (care II protocol). Cephalalgia (Epub)

27. Grazzi L, Andrasik F, Usai S, Bussone G (2008) In-patient vs. day-hospital withdrawal treatment for chronic migraine with medication overuse and disability assessment: results at one-year follow-up. Neurol Sci 29:S161-S163

28. Scher AI, Midgette LA, Lipton RB (2008) Risk factors for headache chronification. Headache 48:16-25

29. Diener HC, Bussone G, van Oene JC, Lahaye M, Schwalen S, Goadsby PJ (2007) Topiramate reduces headache days in chronic migraine: a randomized, double-blind, placebo-controlled study. Cephalalgia 27:814-823 